\title{
Parkin regulates drug-taking behavior in rat model of methamphetamine use disorder
}

\author{
Akhil Sharma', Arman Harutyunyan,2, Bernard L. Schneider ${ }^{3}$ and Anna Moszczynska (1)
}

\begin{abstract}
There is no FDA-approved medication for methamphetamine (METH) use disorder. New therapeutic approaches are needed, especially for people who use METH heavily and are at high risk for overdose. This study used genetically engineered rats to evaluate PARKIN as a potential target for METH use disorder. PARKIN knockout, PARKIN-

overexpressing, and wild-type young adult male Long Evans rats were trained to self-administer high doses of METH using an extended-access METH self-administration paradigm. Reinforcing/rewarding properties of METH were assessed by quantifying drug-taking behavior and time spent in a METH-paired environment. PARKIN knockout rats self-administered more METH and spent more time in the METH-paired environment than wild-type rats. Wild-type rats overexpressing PARKIN self-administered less METH and spent less time in the METH-paired environment. PARKIN knockout rats overexpressing PARKIN self-administered less METH during the first half of drug self-administration days than PARKIN-deficient rats. The results indicate that rats with PARKIN excess or PARKIN deficit are useful models for studying neural substrates underlying "resilience" or vulnerability to METH use disorder and identify PARKIN as a novel potential drug target to treat heavy use of METH.
\end{abstract}

\section{Introduction}

Methamphetamine (METH) use disorder is a worldwide health problem. In the United States, there are close to 2,000,000 people who use METH, and deaths from METH overdose are rapidly rising ${ }^{1,2}$. Despite numerous clinical trials conducted to date, there is no FDAapproved medication for METH use disorder. The medications tested in clinical trials have shown low efficacy in people who use METH moderately and no effect in those who use METH heavily ${ }^{3-5}$. New therapeutic approaches are needed, particularly for people who use METH heavily, as they suffer the most from METH abuse-related neuropsychological problems ${ }^{6-8}$, are less likely to seek treatment than those using the drug moderately ${ }^{9}$, and are at high risk of dying from METH overdose ${ }^{10}$. Early

\footnotetext{
Correspondence: Anna Moszczynska (amosz@wayne.edu)

${ }^{1}$ Department of Pharmaceutical Sciences, Eugene Applebaum College of

Pharmacy and Health Sciences, Wayne State University, 259 Mack Ave, Detroit, Ml 48201, USA

${ }^{2}$ College of Pharmacy, Natural and Health Sciences, Manchester University,

10627 Diebold Rd, Fort Wayne, IN 46845, USA

Full list of author information is available at the end of the article
}

intervention in METH abuse by lowering METH intake is essential not only for preventing METH overdose but also for subsequent interventions, as greater treatment participation is achieved when METH use is low'.

METH use disorder has been linked to alterations in dopaminergic and glutamatergic neurotransmission, alterations in energy metabolism and cytoskeletal arrangement as well as to oxidative stress and inflammation $^{11-24}$. Protein-ubiquitin ligase PARKIN may be a potential novel drug target in METH use disorder as its function has been linked to these processes ${ }^{25-31}$. Despite evidence for a potential role of PARKIN in METH use disorder, ours is the first study linking PARKIN to this disorder. We used genetically engineered rats to evaluate PARKIN as a potential target for METH use disorder, namely PARKIN-deficient (PKO), PARKIN-overexpressing (PO), and wild-type (WT) young adult male Long Evans rats. PARKIN was overexpressed in the nucleus accumbens because the nucleus accumbens is involved in multiple phases of the development of compulsive self-administration of 
stimulants, including its initiation, development, and maintenance ${ }^{32}$ and because it is the hub between emotion, motivation, and action ${ }^{33}$.

Drug addiction is a chronic, relapsing disorder that has been characterized by compulsive seeking and escalated intake of drugs. In the current study, were trained rats to self-administer high doses of METH using an extendedaccess METH self-administration (EA METH SA) paradigm with three ratio schedules of reinforcement. This paradigm produced an escalation of METH intake with rats working much harder to obtain METH with an increasing ratio schedule and, therefore, modeled important aspects of human drug abuse (compulsive drug taking, cravings, and dependence $)^{34}$ resulting in heavy consumption of METH seen in some people dependent on METH. Furthermore, similar to people who abuse $\mathrm{METH}$, rats with extended access to intravenous METH demonstrate cognitive deficits ${ }^{35}$. Rats were allowed to self-administer high METH doses for 10 days. Reinforcing properties of METH were assessed by measuring the number of lever presses for METH and METH intake during each of ten operant sessions. Rewarding properties of METH were assessed by measuring time spent in a METH-paired environment. We demonstrate that PKO rats are predisposed to heavy METH use compared to WT counterparts whereas rats overexpressing PARKIN in the nucleus accumbens are less predisposed.

\section{Materials and methods}

\section{Animals}

The study employed young adult male Long Evans rats ( $~ 55$ days old at the beginning of the study, $N=$ 177) of four genotypes: WT rats (Harlan, Indianapolis, IN, USA), PKO rats (Park2 ${ }^{-1-}$, SAGE Labs, Missouri, MO, USA), PO rats, and PKO PO rats. PO rats were generated by bilaterally overexpressing PARKIN in the nucleus accumbens of WT rats whereas PKO PO rats were generated by bilaterally overexpressing PARKIN in the nucleus accumbens of PKO rats. The stereotaxic surgery was performed as previously published ${ }^{36}$, with some modifications. Microinjections were done at a $16^{\circ}$ angle into these coordinates: $+1.8 \mathrm{~A} / \mathrm{P}, \pm 3.2 \mathrm{M} / \mathrm{L},-7.6$ $\mathrm{D} / \mathrm{V}$ from bregma. Validation of PARKIN protein loss in PKO rats is shown in supplementary Fig. S1, whereas validation of PARKIN overexpression is shown in Fig. 1. PARKIN was not present in PKO samples as assessed by sodium dodecyl sulfate-polyacrylamide gel electrophoresis/western blotting. Upon arrival, animals were pair-housed and maintained under standard environmental conditions in an AAALAC-accredited vivarium. Animals were maintained on a $12 \mathrm{~h}$ light/dark cycle with ad libitum access to food and water unless specified otherwise. Bodyweight was monitored on a daily basis before and during experiments (Suppl. Fig. S2). No method of randomization into groups was used. All experiments were approved by the Wayne State University Institutional Animal Care and Use Committee and conducted in compliance with the ARRIVE guidelines.

\section{Extended-access methamphetamine self-administration (EA METH SA) and conditioned place preference test}

In the first experiment, PKO and WT rats were trained to self-administer high doses of METH (measurement of METH reinforcement) (Fig. 1A) or subjected to the conditioned place preference test (measurement of METH reward) (Fig. 2A). In the second experiment, the reinforcing/rewarding properties of METH were assessed in PO rats as compared to WT rats. In the third experiment, PARKIN was overexpressed in the nucleus accumbens of PKO rats that were subsequently compared to non-overexpressing $\mathrm{PKO}$ rats (phenotype rescue experiment). During EA METH SA, rats had access to METH $(0.1 \mathrm{mg} / \mathrm{kg} /$ injection) for $15 \mathrm{~h} /$ day for 10 days at increasing fixed ratio (FR) schedule: FR1 (days 1-3), FR2 (days 4-6), and FR5 (days 7-10). During METH conditioning for conditioned place preference test, rats were injected with $4 \mathrm{mg} / \mathrm{kg} \mathrm{METH}$ or saline on alternate days.

Supplementary Online Data provides extensive details on stereotaxic surgery as well as the EA METH SA and conditioned place preference.

\section{Electrophoresis and western blotting}

Rats were sacrificed 10 days after the last operant session. Nucleus accumbens was punched out of $2 \mathrm{~mm}$-thick coronal brain section encompassing $0.7-2.7 \mathrm{~mm}$ from bregma. Electrophoresis and western blotting on nucleus accumbens pieces were performed as previously described $^{37}$, utilizing anti-PARKIN and anti- $\beta$-ACTIN (1:1000, 2132S, and 3700S, respectively, Cell Signaling Technology, Danvers, MA, USA) primary antibodies. Immunoreactivities were quantified using ImageJ software (National Institutes of Health, Bethesda, MD, USA) and presented as relative optical densities normalized to control samples.

\section{Statistical analyses}

The experimental data sets were analyzed using IBM SPSS v.25 (IBM, Armonk, NY). EA METH SA data were analyzed by two-way mixed-design ANOVA whereas METH conditioned place preference data were analyzed by two-way factorial ANOVA, both with Bonferroni correction. Before each ANOVA, the data were examined for the presence of outliers using an IQR of 3. After the removal of outlier(s) the data were examined for normality (Shapiro-Wilk's test), equality of variances (Levene's test), and sphericity (Mauchly's test). When Mauchly's test was 
A Timeline for Experiments with Parkin Overexpression

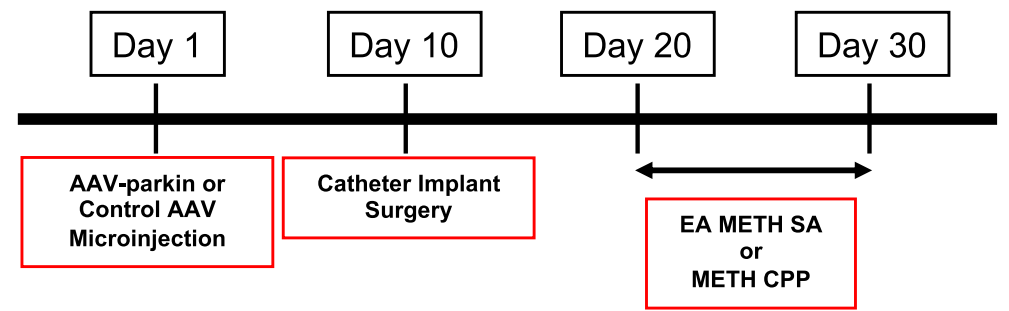

B Parkin Overexpression Site

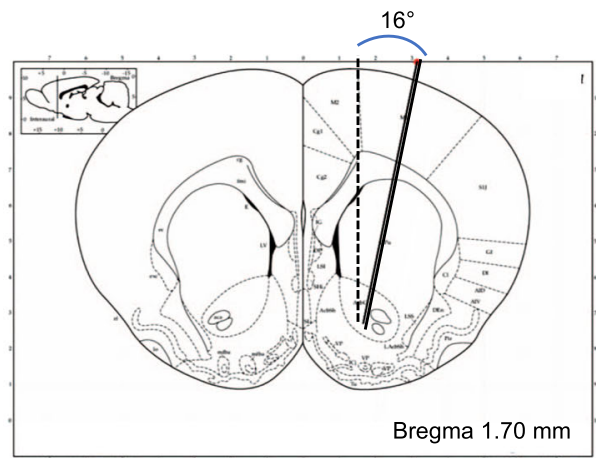

C

\section{Parkin Overexpression Volume}

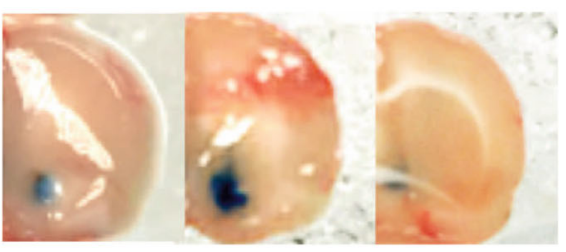

From bregma

\section{Parkin Overexpression Dose Response}
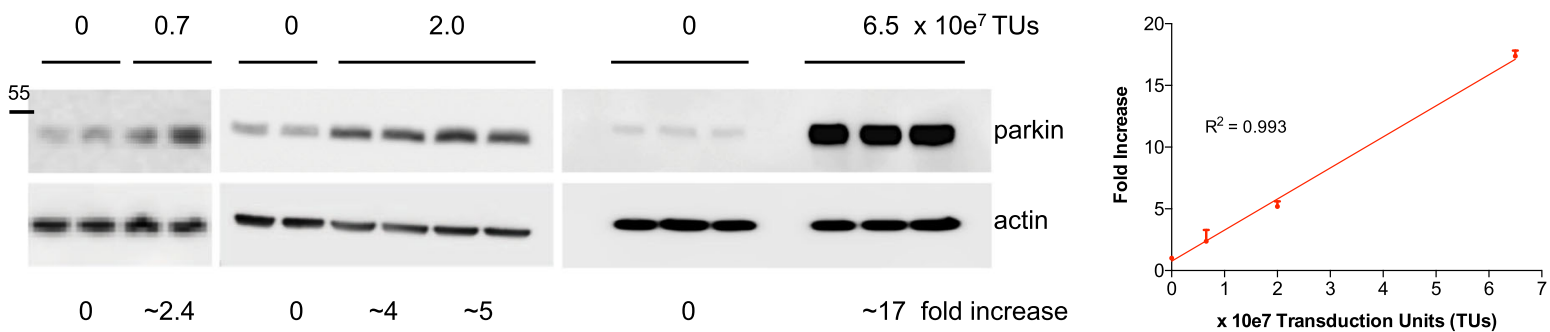

E

Parkin Overexpression Time Course
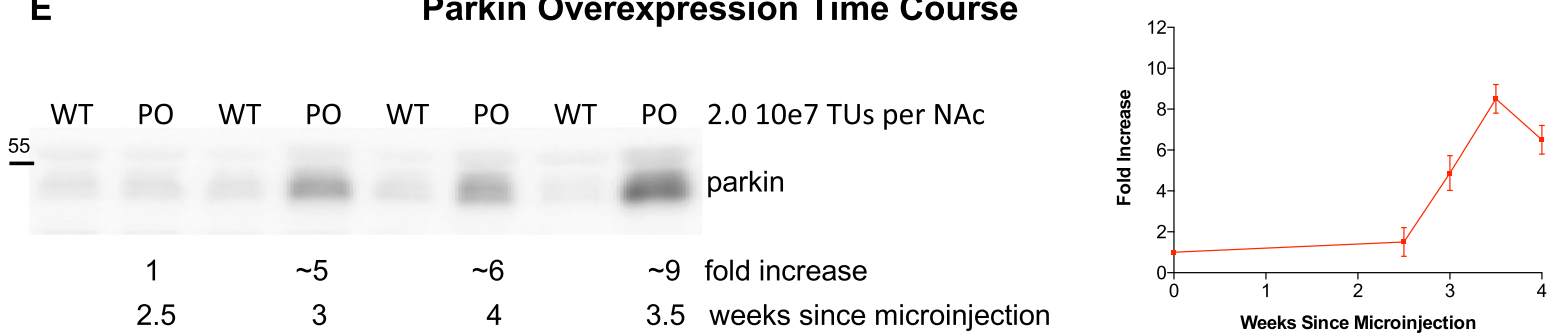

Fig. 1 Validation of PARKIN overexpression. A Timeline for experiments with PARKIN overexpression. B, C Validation of the overexpression site (nucleus accumbens, $0.7-2.7 \mathrm{~mm}$ from bregma) and spread (medial-lateral, dorsal-ventral, and anterior-posterior). D Dose-response of PARKIN overexpression $\left(0,0.7,2\right.$, or $\left.6.5 \times 10^{7} \mathrm{TU} / \mathrm{side}\right)$ in the nucleus accumbens $\left(n=3\right.$ or $4 /$ group). The data followed a linear regression curve $\left(R^{2}=0.993\right.$, $p<0.0001)$. E Time course of PARKIN overexpression $\left(2 \times 10^{7} \mathrm{TUs} / \mathrm{side}\right)$ showing that PARKIN overexpression reached the maximum at around 3.5 weeks after the gene transfer vector microinjection and excess of PARKIN persisted at least for the duration of the study ( 4 weeks) (D). Parkin band migrated to $\sim 52 \mathrm{kDa}$ (relate to the marked molecular weight of $55 \mathrm{kDa}$ ). Parkin band was the only band detected; a representative full blot is presented in Fig. 4. 


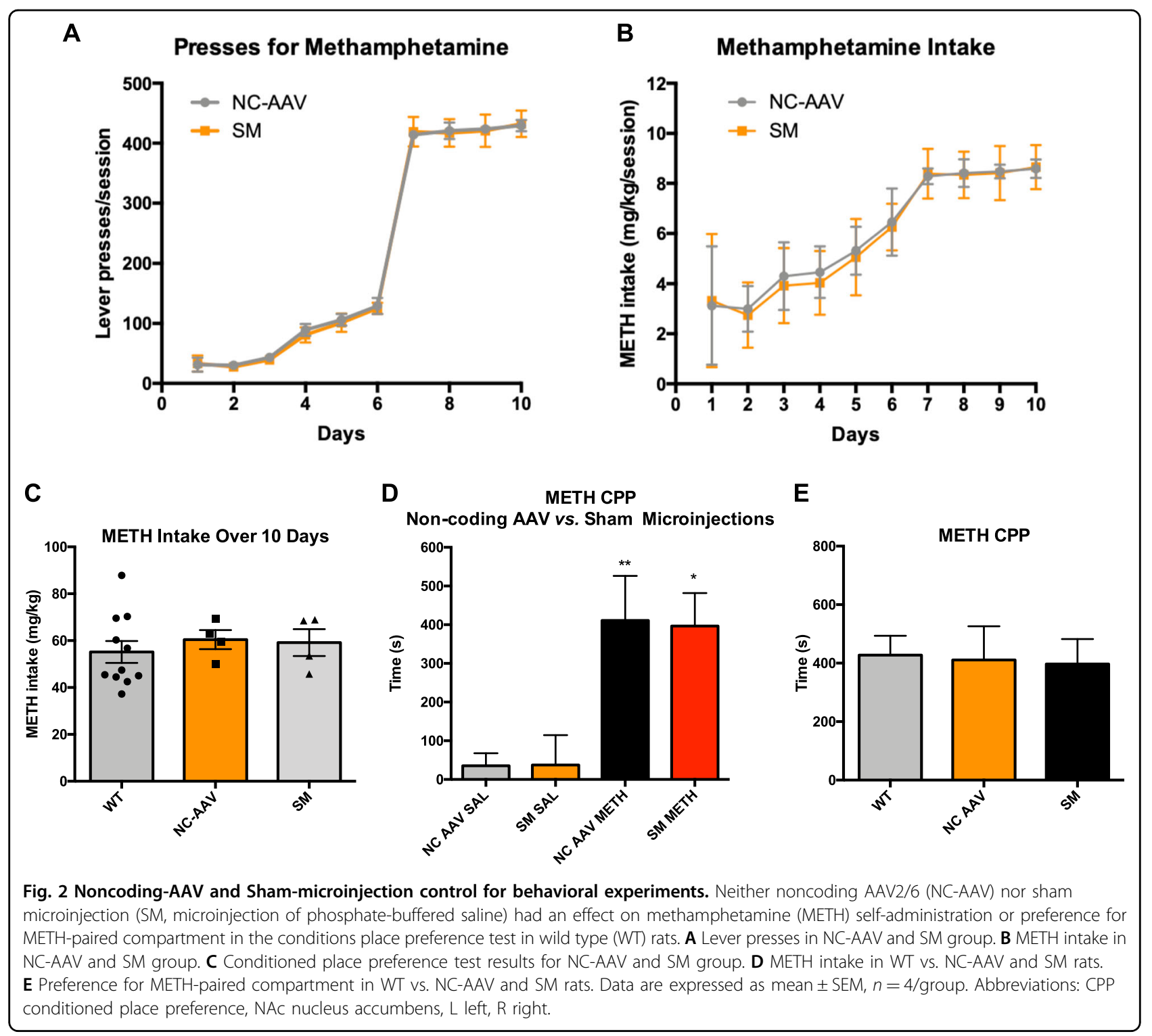

statistically significant, a Greenhouse-Geisser correction was employed. Group differences in PARKIN levels or total METH intake were assessed with the unpaired twotailed Student's $t$ test. Group sizes were estimated based on published behavioral data. To assess sizes of genotypemediated effects and probabilities of the presence of the effects, partial eta square $\left(\eta_{\mathrm{p}}{ }^{2}\right)$ and power of analysis $(1-\beta)$ values were calculated. Statistical significance was set at $p \geq 0.05$. Pearson's correlation analysis was performed on PARKIN overexpression data. Body weight data were analyzed by repeated measure ANOVA with Bonferroni post hoc test. All data, with the exception of body weight data, are reported as mean \pm SEM. Body weight data are reported as mean \pm SD (Supplementary data, Fig. S2). The investigators were not blinded to the group allocation during the experiments or when assessing the outcome.

\section{Results}

\section{Validation of PARKIN overexpression}

Viral vector microinjections took place 3 weeks before METH self-administration or conditioned place preference experiments to allow for maximal PARKIN overexpression (Fig. 1A). Investigation of the microinjection sites using Trypan blue dye confirmed that during microinjections, the needle reached approximately the middle of the nucleus accumbens (Fig. 1B) and that $2 \mu \mathrm{L}$ of the dye did not spread beyond the borders of the nucleus accumbens (Fig. 1B, C). Administration of $0.7 \times 10^{7}, 2 \times$ $10^{7}$, or $6.5 \times 10^{7} \mathrm{TUs} /$ side of AAV2/6-parkin consistently produced a $1.8-3,4-6$, or 15-20-fold increase, respectively, in PARKIN levels in WT rats; the data followed linear regression curve $\left(R^{2}=0.993, p<0.0001\right)$ (Fig. 1D). PARKIN overexpression reached the maximum at around 
3.5 weeks after the gene transfer vector microinjection and lasted till the end of the behavioral experiments ( $\sim 4$ weeks) (Fig. 1E).

We next assessed whether noncoding AAV2/6 or sham microinjection (SM) of saline has an effect on the outcome of EA METH SA or METH conditioned place preference test. Rats were bilaterally microinjected with noncoding AAV2/6 or $2 \mu \mathrm{L}$ sterile phosphate-buffered saline into the nucleus accumbens. These groups were named noncodingAAV (NC-AAV) group and SM group, respectively. Both groups underwent EA METH SA and METH conditioned place preference test. During METH self-administration, both NC-AAV and SM rats pressed for METH at a similar rate (main effect of microinjection type (noncoding AAV2/ 6 or saline): $F_{(1,6)}=0.015, p=0.908, \eta_{\mathrm{p}}{ }^{2}=0.002,1-\beta=$ 0.051; main effect of time: $F_{(9,54)}=101, p<0.001, \eta_{\mathrm{p}}{ }^{2}=$ $0.994,1 \beta=1.0 ; n=4$ /group) (Fig. 2A). Concordant with this result, neither microinjection of noncoding AAV2/6 nor microinjection of saline significantly influenced METH intake (main effect of microinjection type: $F_{(1,6)}=0.033$, $p=0.862, \eta_{\mathrm{p}}^{2}=0.005,1-\beta=0.053$; main effect of time: $F_{(9,54)}=62.2, \quad p<0.001, \quad \eta_{\mathrm{p}}{ }^{2}=0.912, \quad 1-\beta=1.0 ; \quad n=4 /$ group) (Fig. 2B). Total consumption of METH (over 10 days of EA METH SA) by WT, NC-AAV, and SM group is presented in Fig. 2C.

During the conditioned place preference test, both NCAAV and SM rats displayed a preference for the METHpaired compartment (main effect of treatment: $F_{(1,12)}=$ 19.6, $p<0.001, \eta_{\mathrm{p}}{ }^{2}=0.620,1-\beta=0.982, n=4 /$ group; NCAAV: $F_{(1,12)}=10.2, p<0.01, \eta_{\mathrm{p}}{ }^{2}=0.460,1-\beta=0.835$; SM: $\left.F_{(1,12)}=9.36, p<0.05, \eta_{\mathrm{p}}{ }^{2}=0.438 .1-\beta=0.802\right)$ and did not differ in respect to time spent in this compartment $(p>0.1)$ (Fig. 2D). Similarly, these experimental groups did not significantly differ in respect to time spent in the saline-paired compartment $(p>0.1)$. There was no statistically significant interaction between treatment and microinjection type on METH-paired place preference $(p>0.1)$. Comparison of conditioned place preference data for WT, NC-AAV, and SM groups is presented in Fig. 2E. In summary, the NC-AAV and SM groups did not differ from non-microinjected WT rats in respect to selfadministered METH or degree of preference for the METH-paired compartment. This data indicated that neither noncoding AAV2/6 nor SM influenced the outcomes of EA METH SA or METH conditioned place preference test.

\section{PARKIN knockout increases extended-access METH self- administration}

To determine whether PKO rats are more dependent on METH as compared to WT rats, the rats from both genotypes were subjected to the EA METH SA test (Fig. 3A). Both WT and PKO rats showed an escalation of METH selfadministration (Fig. 3B, C) The PKO rats weighed more than
WT rats but lost weight at the same rate as WT rats during EA METH SA (Fig. S2A). Including body weight as a covariate in ANOVA analysis showed that it did not influence METH self-administration $\left(F_{(1,19)}=0.100, p=0.756, \eta_{\mathrm{p}}{ }^{2}=\right.$ $0.005,1-\beta=0.06, n=11$ /genotype). There was a significant main effect of genotype $\left(F_{(1,20)}=16.7, p<0.01, \eta_{\mathrm{p}}{ }^{2}=0.454\right.$, $1-\beta=0.973)$ as well as time $\left(F_{(2.9,58)}=322, p<0.001, \eta_{\mathrm{p}}{ }^{2}=\right.$ $0.942,1-\beta=1.0)$ on lever presses for METH, and significant interaction between the two factors $\left(F_{(2.9,58)}=5.40, p<0.01\right.$, $\left.\eta_{\mathrm{p}}{ }^{2}=0.213,1-\beta=0.913\right)$. Pairwise comparisons revealed that PKOs pressed more than WTs during the first two sessions $(p<0.01$ and $p<0.001$, respectively) and during the compulsive phase of METH self-administration (day 7, $p<0.05$ and days $8-10, p<0.01$ ) (Fig. 3B). Concordant with this result, genotype and time both significantly influenced METH intake (genotype: $F_{(1,20)}=13.8 . p<0.01, \eta_{\mathrm{p}}{ }^{2}=0.409$, $1-\beta=0.941$; time: $F_{(3.6,71)}=53.8, p<0.001, \eta_{\mathrm{p}}{ }^{2}=0.729,1-\beta=$ $1.0)$, but there was no significant interaction between the two factors $\left(F_{(3.6,71)}=1.40, p=0.246\right)$. The pairwise comparisons revealed that $\mathrm{PKO}$ rats self-administered significantly more METH during the first two sessions $(p<0.01$ and $p<0.001$, respectively) and during the compulsive phase of METH selfadministration (day $7, p<0.05$ and days $8-10, p<0.01$ ) (Fig. $3 \mathrm{C})$. There was no significant difference between PKO and WT rats in respect to the number of presses for saline (Fig. 3B inset, $n=4$ /genotype), confirming that METH, not the light stimulus, acted as a reinforcer. The total amount of METH consumed by PKO rats was significantly higher than that consumed by WT rats $(+39 \%, p<0.001)$ (Fig. 3D).

The results from the EA METH SA indicate that METH exerts stronger reinforcing effects in rats lacking PARKIN than in WT controls. The effect of PARKIN knockout on METH self-administration was of large size (large $\eta_{\mathrm{p}}{ }^{2}$ ) and significant, thus strongly suggesting PARKIN as a suppressor of reinforcing effects of METH.

\section{PARKIN knockout increases preference for METH-paired compartment}

Compulsive drug use is maintained by the rewarding properties of the drug and by the loss of control over drug intake. To determine whether PARKIN knockout increases the rewarding effects of METH, PKO, and WT rats underwent the conditioned place preference test (Fig. 3E). Though the conditioned place preference test has limitations $^{38}$, it provides information about the rewarding effect of contextual cues associated with a drug stimulus. Twoway ANOVA revealed a trend for significant interaction between genotype and treatment $\left(F_{(1,20)}=3.31, p=0.084\right.$, $\left.\eta_{\mathrm{p}}{ }^{2}=0.142,1-\beta=0.410\right)$, and main effects of both genotype and treatment on METH preference, with the effect of treatment being stronger than the effect of genotype $\left(F_{(1,20)}=79.2, \quad p<0.001, \quad \eta_{\mathrm{p}}{ }^{2}=0.798, \quad 1-\beta=1.00, \quad\right.$ and $F_{(1,20)}=4.50, p<0.05, \eta_{\mathrm{p}}{ }^{2}=0.184,1-\beta=0.523$, respectively, $n=6$ /group). Pairwise comparisons revealed that 


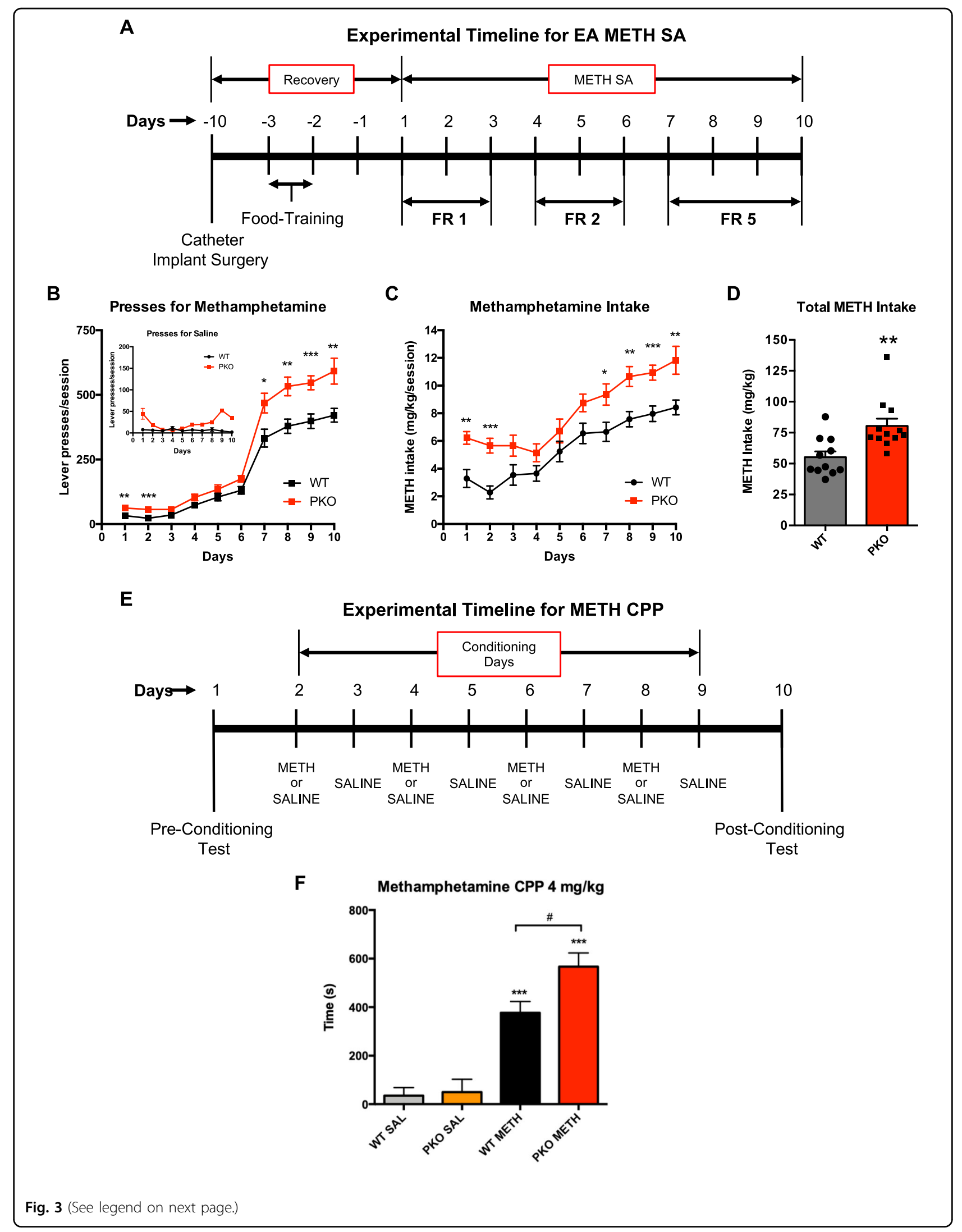


(see figure on previous page)

Fig. 3 PARKIN knockout increases extended-access methamphetamine self-administration (EA METH SA) and METH reward. A Timeline of EA METH SA experiment. B, C Escalation of METH taking in Parkin gene knockout (PKO) rats as compared to wild-type (WT) rats during an increasing ratio schedule of reinforcement: FR1, FR2, and FR5. The PKO rats pressed for METH at a significantly higher rate than the WT rats (B) with a consequent higher consumption of METH (C). There was no significant difference between PKO and WT rats in respect of the number of presses for saline (B inset, $n=4$ /group). ${ }^{*} p<0.05,{ }^{* *} p<0.01,{ }^{* * *} p<0.001, n=11$ group. $\mathbf{D}$ Total amount of METH consumed by PKO rats was significantly higher than that consumed by WT rats $\left(+39 \%{ }^{* *} p<0.001\right)$. E Timeline of conditioned place preference experiment. $\mathbf{F}$ The PKO rats displayed a significantly higher preference for METH ( $4 \mathrm{mg} / \mathrm{kg})$ than the WT rats. ${ }^{* * *} p<0.001$ as compared to the respective saline (SAL) controls, ${ }^{*} p<0.05 \mathrm{WT}$ vs. PKO, $n=6 /$ group. Data are expressed as mean \pm SEM. Abbreviations: CPP conditioned place preference, $F R$ fixed ratio.

there was no significant difference in baseline preference for a compartment between PKO and WT rats $(p=0.834)$ (Fig. 3F). Both genotypes showed a significant METHinduced conditioned place preference $(p<0.001)$, with the PKO rats displaying a significantly higher preference for the METH-paired compartment than the WT rats $(p<$ 0.05) (Fig. 3F). ANCOVA determined that bodyweight had no influence on the results (not shown).

The results from the conditioned place preference test suggest that METH-paired contextual cues are more rewarding for PKO rats than for WT rats.

\section{Extended-access METH self-administration leads to PARKIN deficit in the nucleus accumbens}

We previously determined that METH binge decreases PARKIN levels in the striatum via oxidative damage ${ }^{39}$. To determine whether exposure of WT rats to the EA METH SA paradigm decreases PARKIN levels in the nucleus accumbens, PARKIN levels were assessed in rats that selfadministered METH at 10 days after the last operant session and compared to PARKIN levels in rats exposed to saline. METH-exposed rats had significantly lower levels of PARKIN in the nucleus accumbens as compared to saline-exposed rats $(-24 \%, t=3.02, d f=15, p<0.01$, $n=8$ or 9$)$ (Fig. 4 A, B).

\section{WT rats overexpressing PARKIN self-administer less METH and spend less time in the METH-paired compartment than WT controls}

Since WT rats subjected to EA METH SA had lower PARKIN levels in the nucleus accumbens than saline controls, we next investigated whether overexpression of PARKIN in the nucleus accumbens of WT rats would result in attenuated METH self-administration as compared to non-overexpressing WT rats. Two concentrations of AAV2/6-parkin were utilized to overexpress PARKIN$2 \times 10^{7}$ or $6.5 \times 10^{7} \mathrm{TUs} /$ side (usulally producing $4-6$ or 15-20-fold excess of PARKIN). The PO rats weighed slightly less than WT controls (Fig. S2B); however, body weight did not have a significant effect on METH selfadministration as a covariate $\left(F_{(1,9)}=0.006, p=0.939\right.$, $\eta_{\mathrm{p}}{ }^{2}=0.001,1-\beta=0.051, n=6$ /group). At low levels of PARKIN overexpression, there was a significant main effect of time $\left(F_{(3.1,31.5)}=51.6, p<0.001, \eta_{\mathrm{p}}{ }^{2}=0.838\right.$, $1-\beta=1.0)$, but not of genotype $(p>0.1)$, on lever presses for METH (Fig. 5A). There was no statistically significant interaction between the two factors $(p>0.1)$. Statistical analysis of METH intake over 10 days produced a similar result: there was a significant effect of time $\left(F_{(1.54,15.4)}=\right.$ 187, $\left.p<0.001, \eta_{\mathrm{p}}{ }^{2}=0.949,1-\beta=1.0\right)$, but no significant main effect of genotype $(p>0.1)$ and no significant genotype $\times$ time interaction $(p>0.1)$ (Fig. 5B). Ten days after the last operant session, the average PARKIN overexpression in PO-L group was 3.8-fold (not shown). There was no statistically significant correlation between total METH intake and PARKIN levels $\left(R^{2}=0.077, p=0.382\right)$.

At high levels of PARKIN overexpression, there was a strong significant effect of genotype $F_{(1,10)}=47.8, p<$ $\left.0.0001, \eta_{\mathrm{p}}{ }^{2}=0.827,1-\beta=1.0\right)$, as well as time $\left(F_{(2.19,21.9)}=\right.$ 543, $\left.p<0.0001, \eta_{\mathrm{p}}{ }^{2}=0.982,1-\beta=1.0\right)$, on METH lever pressing (Fig. $5 \mathrm{C}$ ) and significant interaction between these variables $\left(F_{(2.19,21.9)}=34.9, p<0.0001, \eta_{\mathrm{p}}{ }^{2}=0.577\right)$. Pairwise comparisons revealed that $\mathrm{PO}$ rats pressed significantly less for METH on days 4-10, with the strongest effect at the compulsive stage of METH abuse (day $4, p<$ 0.001 ; days 5 and $6, p<0.05$; day $7 p<0.001$; days $8-10, p<$ 0.0001 ) (Fig. $5 \mathrm{C}$ ). In concordance, there was a strong significant effect of genotype $\left(F_{(1,10)}=31.6, p<0.0001, \eta_{\mathrm{p}}{ }^{2}=\right.$ $0.760,1-\beta=0.999)$ as well as time $\left(F_{(4,40)}=103, p<0.0001\right.$, $\left.\eta_{\mathrm{p}}{ }^{2}=0.912,1-\beta=1.0\right)$ on METH intake and significant interaction between these variables $\left(F_{(4,40)}=14.4, p<\right.$ $\left.0.0001, \eta_{\mathrm{p}}{ }^{2}=0.573\right)$. As with lever presses, pairwise comparisons showed that POs took significantly less METH on days $4-10$, with the strongest effect on days $8-10$ (day 4 , $p<0.001$; days 5 and $6, p<0.05$; day $7, p<0.001$; days $8-10$, $p<0.0001$ ) (Fig. 5D). Figure 5E presents quantification of total METH intake in PO-L and PO-H rats as compared to their respective WT control groups. There was no significant difference in total METH intake between the PO-L group and WT controls, whereas the PO-H group consumed significantly less METH than the corresponding WT controls $\left(-33 \%,{ }^{* * * *} p<0.001\right)$. Ten days after the last operant session, the average PARKIN overexpression in the PO-H group was 16-fold (6-19 fold) (Fig. 5F). Pearson's correlation analysis revealed that total METH intake by the PO-H group highly and significantly correlated with PARKIN levels in the nucleus accumbens $\left(R^{2}=0.831, p<\right.$ 0.0001) (Fig. 5F). 
A

SAL METH METH SAL SAL METH METH METH

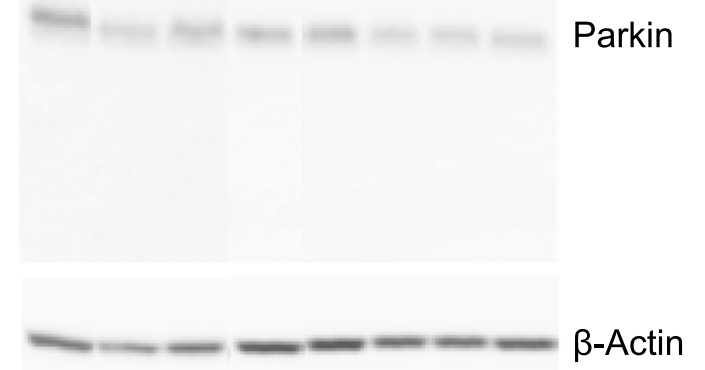

B

B Parkin Levels in the NAc

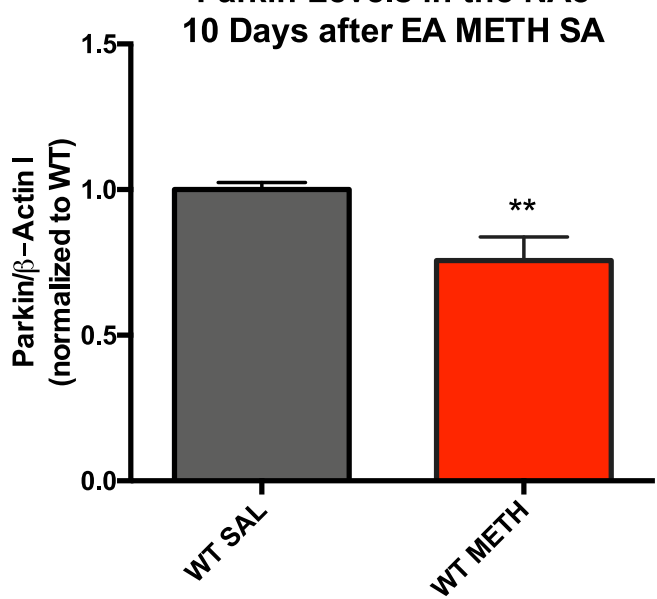

Fig. 4 Extended-access methamphetamine self-administration (EA METH SA) decreases PARKIN protein levels in the nucleus accumbens. A Representative PARKIN bands $(\sim 52 \mathrm{kDa})$ assessed in nucleus accumbens (NAC) of wild type (WT) rats, with $\beta$-ACTIN serving as a loading control: SAL (black) represents saline-yoked WT rats while METH (red) represents WT rats that underwent EA METH SA; both group of rats were killed 10 days after the last operant session. B Quantification of PARKIN bands. As compared to saline (SAL) administration, EA METH SA led to a deficit $(-24 \%)$ in PARKIN levels in the nucleus accumbens of WT rats at 10 days after the last operant session. ${ }^{* *} p<0.01, n=8$ or 9/group. Data are expressed as mean \pm SEM.

WT rats overexpressing PARKIN at high levels spent less time in the METH-paired compartment in the conditioned preference test than non-overexpressing WT controls (Fig. 5G). Two-way ANOVA revealed the main effect of treatment on METH preference $\left(F_{(1,20)}=17.1, p<0.001\right.$, $\left.\eta_{\mathrm{p}}{ }^{2}=0.461,1-\beta=0.976\right)$ and a trend for significant interaction between genotype and treatment $\left(F_{(1,20)}=3.47\right.$, $\left.p=0.077, \eta_{\mathrm{p}}^{2}=0.148,1-\beta=0.426\right)$. Pairwise comparisons detected no significant difference in baseline preference for a compartment between PO and WT rats $(p=0.800, n=6$ / group). Significant preference for the METH-paired compartment, as compared to the saline-paired compartment, was detected in WT rats $(p<0.0001)$ but not in PO rats $(p=0.123)$, however, there was a difference between genotypes in the METH group $(p<0.05)$ (Fig. 5G). The ANCOVA analysis determined that bodyweight had no influence on the results (not shown).

These results further support the conclusion that PARKIN can negatively regulate reinforcing/rewarding properties of METH if its levels are increased at least sixfold.

\section{PARKIN knockout rats overexpressing PARKIN self-} administer less METH during the first half of drug selfadministration compared to PARKIN-deficient rats

The next experiment aimed to determine whether overexpression of PARKIN in the nucleus accumbens of $\mathrm{PKO}$ rats would attenuate METH self-administration as it did in the nucleus accumbens of WT rats. High levels (15-20-fold) of PARKIN were overexpressed in the nucleus accumbens of PKO rats ( $n=5$ or 6 /group), while control rats were microinjected with saline (SM surgery). PKO and PKO PO rats did not differ in respect to body weight during the 10 days of METH SA (Fig. S2C). There was a significant main effect of time $\left(F_{(1.2,11)}=192, p<0.0001\right.$, $\left.\eta_{\mathrm{p}}{ }^{2}=0.838,1-\beta=1.0\right)$ and a weaker main effect of genotype $\left(F_{(1,9)}=5.15, p=0.05, \eta_{\mathrm{p}}{ }^{2}=0.364,1-\beta=0.525\right)$ on lever presses for METH between the genotypes (Fig. 6A). There was no statistically significant interaction between the time and genotype $(p>0.1)$. Examination of METH intake data produced similar results: a significant main effect of time $\left(F_{(1.5,13.4)}=29.3, p<0.00010 .765,1-\beta=1.0\right)$ and genotype $\left(F_{(1,9)}=18.3, p<0.01, \eta_{\mathrm{p}}{ }^{2}=0.671,1-\beta=\right.$ $0.966)$ but no significant genotype $\times$ time interaction $(p>$ 0.1). Pairwise comparisons revealed that PKO PO rats pressed significantly less for METH and, consequently, ingested significantly less of the drug than nonoverexpressing PKO rats in the first half of EA METH SA (days 1,4 , and 5) $(p<0.01, p<0.01$, and $p<0.001$, respectively) but not at later stages of EA METH SA (Fig. 6B). The total amount of METH consumed by PKO PO rats was significantly lower than the total amount of METH consumed by PKO rats $(-18 \%, p<0.01)$ (Fig. 6C). There was no statistically significant correlation between total METH intake by the PKO PO group and PARKIN levels in the nucleus accumbens measured at 10 days after the last operant session $\left(R^{2}=0.109, p=0.295\right)$ (Fig. 6D).

This result suggests that PARKIN knockout-induced compensatory changes may have lessened the effect of PARKIN overexpression. 


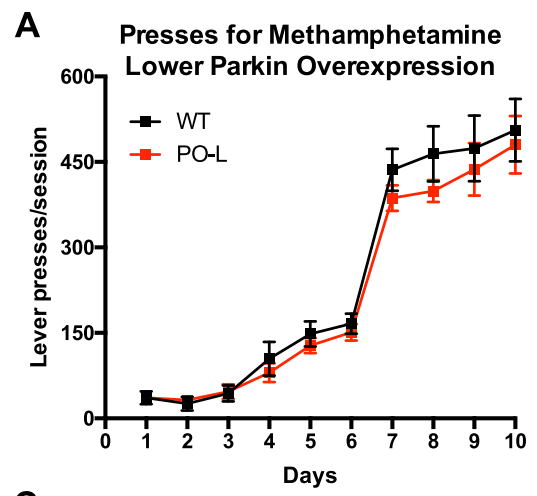

C

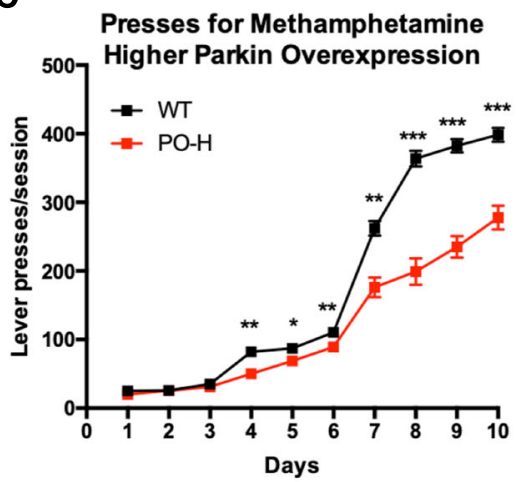

E

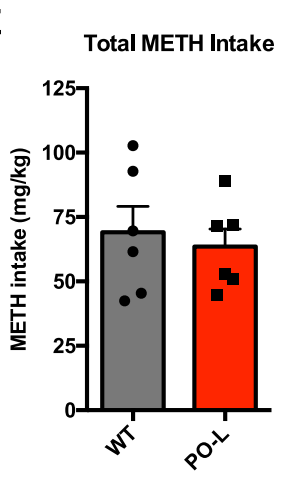

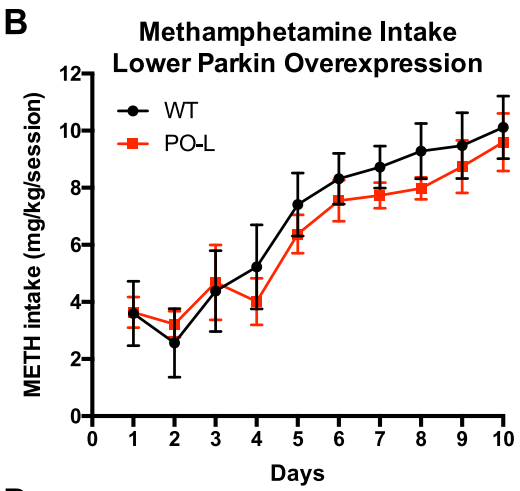

D

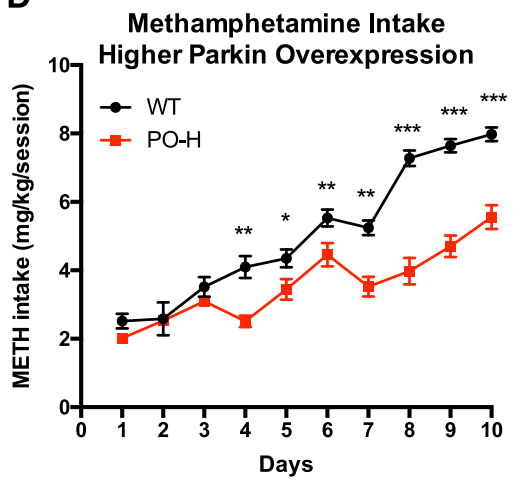

$\mathbf{F}$

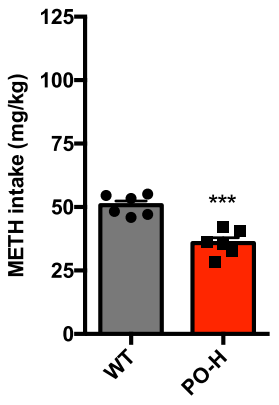
Parkin Overexpression

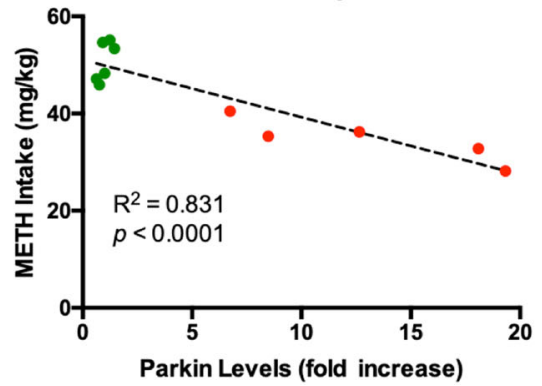

Methamphetamine Intake vs.

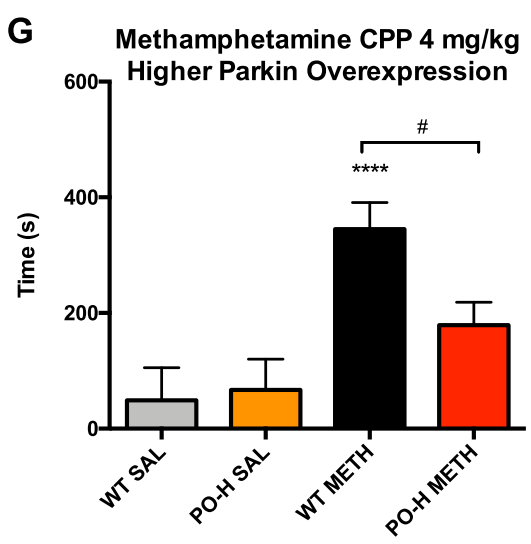

Fig. 5 (See legend on next page.) 
(see figure on previous page)

Fig. 5 PARKIN overexpression in the nucleus accumbens of WT rats attenuates extended-access methamphetamine self-administration (EA METH SA) and METH reward. Escalation of METH taking in rats overexpressing PARKIN in the nucleus accumbens as compared to nonexpressing wild-type (WT) rats during an increasing ratio schedule of reinforcement: FR1, FR2, and FR5. A At the low PARKIN overexpression (4-6fold), the PARKIN overexpressing (PO) rats did not press for METH at a significantly lower rate than the WT rats and, consequently, B they did not consume less METH than the WT rats. C At the high PARKIN overexpression (15-20-fold), the PO rats did press for METH at a significantly lower rate than the WT rats and $\mathbf{D}$ consumed less METH than the WT rats. ${ }^{*} p<0.05,{ }^{* *} p<0.01,{ }^{* * *} p<0.001$, WT vs. PO-H, $n=6 /$ group. E Quantification of total METH intake in PO-L and PO-H rats as compared to their respective WT control groups. There was no significant difference in total METH intake between the PO-L group and WT controls whereas the PO-H group consumed significantly less METH than the WT group $\left(-33 \%\right.$, $\left.{ }^{* * *} p<0.001\right)$. F Correlation analysis of total METH intake with PARKIN levels in PO-H group (red dots: PO-H group; green dots: WT group). G The 15-20-fold excess of PARKIN was sufficient to significantly attenuate preference for METH $(4 \mathrm{mg} / \mathrm{kg})$ in WT rats in the conditioned place preference test. ${ }^{* * *} p<0.001$ as compared to the respective saline controls, ${ }^{*} p<0.05$ WT vs. PO-H, $n=6 /$ group. Data are expressed as mean \pm SEM. Abbreviations: NAc nucleus accumbens, FR fixed ratio, PO-L lower PAKIN overexpression, PO-H higher PARKIN overexpression, SAL saline.

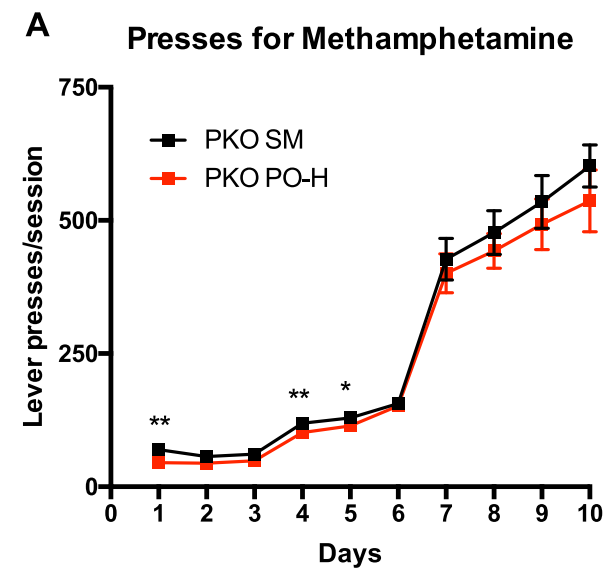

C

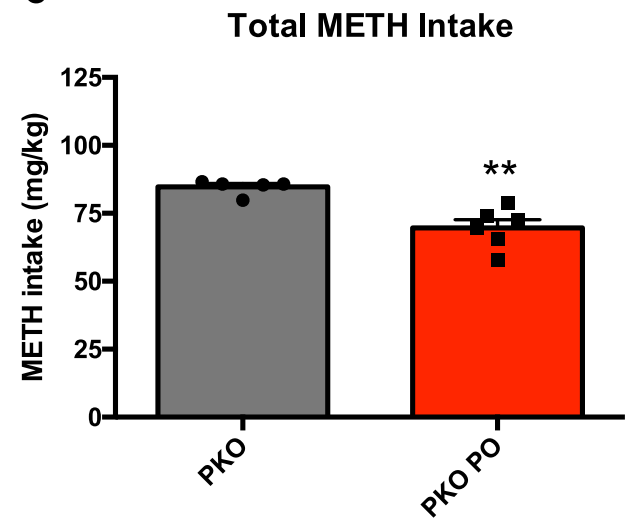

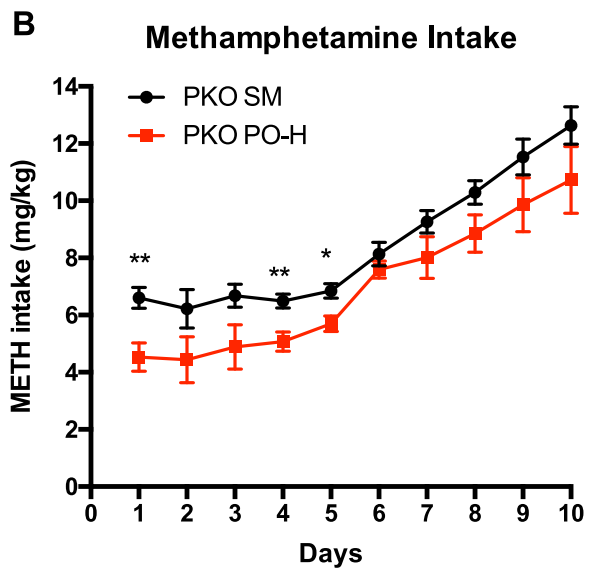

D

Dethamphetamine Intake vs. Parkin Overexpression

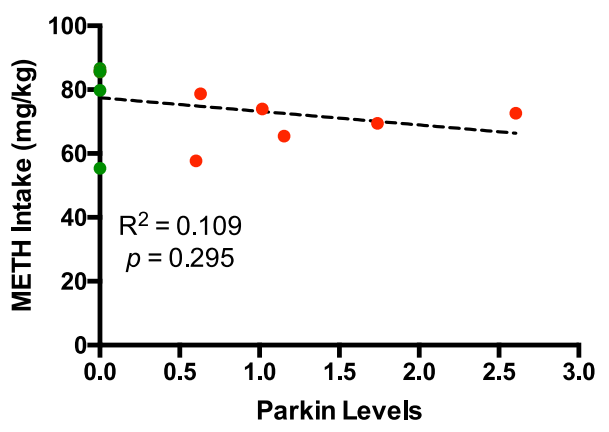

Fig. 6 The effect of PARKIN overexpression in the nucleus accumbens of Parkin gene knockout (PKO) rats. High PARKIN Overexpression (15-20-fold) in the nucleus accumbens of Parkin knockout PKO rats significantly attenuated methamphetamine (METH) taking in the first half of the extended-access METH self-administration as compared to non-overexpressing PKO rats. A Lever presses for METH, B METH intake. C Total amount of METH consumed by PKO PO rats was significantly lower than the total amount of METH consumed by PKO rats ( $-18 \%)$. D Correlation analysis of total METH intake with PARKIN levels in PKO PO group (red dots: PKO PO group; green dots: PKO group). ${ }^{*} p<0.05,{ }^{* *} p<0.01, n=5$ or $6 /$ group. Data are expressed as mean \pm SEM.

\section{Discussion}

METH use disorder is a disorder without pharmacotherapy and, therefore, in need of new drug targets for the development of new drugs. In this study, we used genetically engineered rats to evaluate PARKIN as a potential drug target for METH use disorder. We found that PARKIN knockout rats self-administered more METH and spent more time in the METH-paired environment than WT rats whereas WT rats overexpressing PARKIN in the nucleus accumbens at least sixfold 
self-administered less METH and spent less time in the METH-paired environment. We also found that PARKIN knockout rats overexpressing PARKIN in the nucleus accumbens self-administered less METH during the first half of drug self-administration days than PARKINdeficient rats. The results indicate that rats with PARKIN excess or PARKIN deficit are useful models for studying neural substrates underlying "resilience" or vulnerability to METH use disorder and identify PARKIN as a novel potential drug target to treat heavy use of METH.

\section{The role of PARKIN in METH-taking behavior}

Drug-taking behavior is a consequence of reinforcing effects of the drug of abuse, mediated by goal-directed and habit systems in the brain. At the initial stages of drug self-administration, the goal-directed system directs the relationship between action (lever pressing) and outcome (drug infusion and experiencing sought-after drug effects). After prolonged drug-taking, instrumental action becomes compulsive and habitual ${ }^{40}$. Escalation of drug self-administration is a hallmark of compulsive drug taking and drug dependence ${ }^{34}$. Hence, escalation of METH self-administration by both PKO and WT rats despite the increasing effort required to obtain METH (from 1 to 5 presses needed to obtain METH injection) suggests increasing motivation to obtain the drug, leading to METH dependence. Compared to the WT controls, the PKO rats pressed significantly more for METH and, consequently, consumed more METH than WT rats during the initial stage (FR1) and during the FR5 stage (compulsive intake) of EA METH SA. In contrast, the PO rats pressed significantly less for METH and consumed more of the drug at these stages. Furthermore, METH intake highly correlated with the levels of PARKIN in the nucleus accumbens of WT rats overexpressing PARKIN 6-19-fold. Two conclusions can be reached based on these findings. First is that PARKIN may be involved in negative modulation of reinforcing/rewarding effects of METH within the nucleus accumbens, which is the main brain region mediating rewarding/reinforcing effects ${ }^{41}$. This conclusion is supported by the results from the conditioned place preference test. The conditioned place preference test and self-administration measure different processes, namely the rewarding effects of a drug through context-drug associative learning vs. reinforcing effects of a drug through operant conditioning, respectively. However, there is reasonable concordance between drugs that produce conditioned place preference and drugs that are self-administered ${ }^{38}$. Because both paradigms involve associative learning (albeit of a different type) ${ }^{38,42}$ and because the nucleus accumbens plays a role in this types of learning ${ }^{40,43-45}$, PARKIN may have a role in processes associating METH effects with the context or operant behavior. In addition to playing a key role in mediating reward, the nucleus accumbens controls goal-directed behavior during instrumental conditioning ${ }^{40,46-49}$. Consequently, the second conclusion is that accumbal PARKIN may negatively regulate goal-directed behavior during METH self-administration. We demonstrated that the volume spread after the microinjections were largely confined to the nucleus accumbens. Therefore, it is unlikely that the difference in METH self-administration observed between PO and WT rats was due to unspecific PARKIN overexpression in the adjacent dorsomedial striatum, which is a key brain area regulating goaldirected behavior ${ }^{40,50-52}$, but rather to overexpression of PARKIN in the nucleus accumbens, which has a role in action selection ${ }^{49}$. Habitual drug-taking is mediated by the dorsolateral striatum ${ }^{40,50,53,54}$ and it is not known whether striatal PARKIN affects this behavior. Overexpression of PARKIN in the nucleus accumbens likely resulted in its excess not only in accumbal perikarya but also in terminals located in brain areas to which the nucleus accumbens projects to ${ }^{36}$. Consequently, PARKIN could have presynaptically modulated METH taking in these brain areas. Even though PKO and PO rats displayed opposite addictive behaviors, molecular mechanisms underlying these behaviors may be different due to compensatory mechanisms that occurred in the brains of PKO but not in PO rats during development ${ }^{55}$. Such changes might explain the smaller effect of PARKIN overexpression on METH taking in PKO PO rats as compared to PO rats. Alternatively, total loss of PARKIN was harder to overcome than the moderate PARKIN deficit $(-24 \%)$ induced by METH SA in WT rats.

\section{Molecular determinants of altered METH-taking behavior in PKO and PO rats}

PARKIN is a ubiquitous ubiquitin-protein ligase with neuroprotective and anti-inflammatory properties ${ }^{56-58}$, mostly known for its link to Parkinson's disease ${ }^{59}$. The canonical function of PARKIN is to polyubiquitinate proteins destined for degradation by proteasome or lysosome (short-lived, misfolded, or damaged proteins) ${ }^{26,60}$. PARKIN also has degradation-independent functions, which it exerts by monoubiquitination of its substrates $^{60}$. To date, about three dozen PARKIN substrates have been identified in various cellular compartments, including mitochondrial, endoplasmic reticulum, and synaptic proteins, that belong to various signaling pathways $^{61,62}$.

In relation to the present investigation, studies in young adult PKO mice (3-4-month-old) reported no change in the levels of striatal dopamine or dopamine receptors as compared to WT littermates ${ }^{63,64}$, with dopamine metabolites reported unchanged or elevated ${ }^{65,66}$. Our previous study in young adult PKO rats (2-month-old) similarly found no change in dopamine or dopamine metabolites. 
PKO rats had normal levels of dopamine transporter; however, PARKIN may modulate dopaminergic neurotransmission via ubiquitination of the transporter and regulation of its trafficking ${ }^{67,68}$. Dopamine D2 receptor has not been identified as a substrate for PARKIN; however, we found that PKO rats had lower levels of the glycosylated form of postsynaptic D2 receptor than WT rats in the dorsal striatum ${ }^{37}$. Low D2/D3 receptor availability in striatal regions, including the nucleus accumbens, is considered a risk factor for METH use disorder because it is a molecular feature of impulsivity; animal, as well as human data, support this notion ${ }^{69,70}$. Furthermore, it has been demonstrated that D2-expressing medium spiny neurons in the nucleus accumbens antagonize the reinforcing and rewarding effects of another psychostimulant, cocaine ${ }^{71}$. Parkin gene knockout results in augmented glutamatergic neurotransmission and altered levels of glutamate receptors as well as $\gamma$-aminobutyric acid $B\left(G_{B B A}\right)$ receptors in mice ${ }^{31}$. This data suggests the involvement of PARKIN protein in the regulation of these receptors at the synapse. In conclusion, PARKIN deficit-mediated alterations in dopaminergic, glutamatergic, and/or GABAergic neurotransmission likely modulate the rewarding/reinforcing effects of METH via their receptors $^{72-77}$.

METH use disorder has been linked to alterations in energy metabolism and cytoskeletal arrangement as well as to oxidative stress and inflammation ${ }^{11-21,78-80}$. For example, METH-induced inflammation in the nucleus accumbens was shown to mediate METH reinforcing properties $^{11,78}$. In the EA METH SA paradigm, large doses of METH were self-administered, likely causing oxidative stress and inflammation in multiple brain areas, including the nucleus accumbens ${ }^{11,81}$. PARKIN function has been linked to energy metabolism, and cytoskeletal arrangement as well as to protection against oxidative stress, and inflammation ${ }^{25,26,29-31}$. Therefore, PARKIN is well-positioned to regulate responses to METH and could have decreased METH intake in rats via any of the aforementioned mechanisms.

Addiction circuitry includes multiple brain areas ${ }^{82}$, all of which have likely undergone neuroadaptive changes in the PKO rats during development ${ }^{55}$. We hypothesize that because of these changes and lack of PARKIN in addiction circuitry, overexpression of PARKIN in the nucleus accumbens only had a smaller effect on METH selfadministration in PKO rats than WT rats. Overexpression of PARKIN in the nucleus accumbens and prefrontal cortex and/or dorsal striatum may be necessary to suppress the addictive phenotype in PKO rats. Data from PKO PO rats suggest that PARKIN has a stronger effect on molecular mechanisms underlying reinforcing properties than on those underlying loss of control over METH intake in these rats. It remains to be determined whether PARKIN overexpression in the nucleus accumbens of PKO rats would have a more pronounced effect on METH self-administration in a paradigm with short access to the drug (e.g., $1 \mathrm{~h}$ ), consequently leading to low METH intake.

\section{Limitations and methodological considerations}

A limitation of using PKO rats is that developmental adaptations to the absence of the Parkin gene may have compensated for an early loss of gene function. As a result, such adaptations may have obscured the analysis of the effects of PARKIN protein per se. Another limitation of our study is different environments in which the PKO and WT rats spent the first 2 months of their lives (different vendors), a factor that can affect gene expression and, consequently, phenotype. Nevertheless, overexpression of PARKIN resulted in behaviors opposite to those observed in PKO rats, thus validating the role of PARKIN in METH self-administration. A high level of PARKIN overexpression was needed for the desired effect, which can be viewed as non-translational. However, since we employed a model of very high METH intake, lower levels of PARKIN overexpression will likely work with models of moderate METH intake.

PARKIN activity is regulated by $\mathrm{Zn}^{2+}$, an array of posttranslational modifications as well as by interactions with a variety of regulatory proteins ${ }^{83,84}$. Several research groups have been working on increasing PARKIN activity in relation to Parkinson's or Alzheimer's disease. These studies range from manipulation of Parkin gene to manipulation of upstream and downstream PARKIN interactors. Some studies focus on relieving autoinhibitory interactions within PARKIN. Our laboratory investigates the possibility of increasing PARKIN levels by manipulation of proteins downstream of PARKIN substrates. Currently, there is no effective PARKIN activator for in vivo use despite these research efforts. The efforts continue and, therefore, increasing PARKIN expression or activity pharmacologically may be possible in near future.

\section{Conclusions}

To date, pharmacological approaches have failed as treatments for METH use disorder. We demonstrated for the first time that PARKIN plays important role in this disorder. Specifically, we demonstrated that PARKIN modulates the rewarding/reinforcing properties of highdose METH. The role of PARKIN in drug use disorder has not been previously studied; therefore, our study is novel. From the public health point of view, the most important finding of this investigation is that overexpression of PARKIN in the nucleus accumbens alone was sufficient to attenuate intake of very high doses of METH by WT rats, making PARKIN a novel potential drug target to treat heavy use of METH. In addition, we 
demonstrated that rats with PARKIN excess or PARKIN deficit are useful models for studying neural substrates underlying "resilience" or vulnerability to METH use disorder.

\section{Acknowledgements}

This research was funded by Wayne State University and partially by NIH/NIDA DA034738 grant. Vector design and production was supported by the Swiss National Science Foundation grants 31003A_120653 and 31003A_135696.

\section{Author details}

'Department of Pharmaceutical Sciences, Eugene Applebaum College of Pharmacy and Health Sciences, Wayne State University, 259 Mack Ave, Detroit, MI 48201, USA. ${ }^{2}$ College of Pharmacy, Natural and Health Sciences, Manchester University, 10627 Diebold Rd, Fort Wayne, IN 46845, USA. ${ }^{3}$ Brain Mind Institute, École Polytechnique Fédérale de Lausanne School of Life Sciences, Ch. Des Mines, 9, CH-1202 Geneve, Switzerland

\section{Author contributions}

A.S. and A.M. analyzed and interpreted the data, prepared the figures, and cowrote the paper. A.H. significantly contributed to the data generation. B.S. provided AAVs and consulted on the PARKIN overexpression. All authors reviewed and edited the paper.

\section{Conflict of interest}

The authors declare no competing interests.

\section{Publisher's note}

Springer Nature remains neutral with regard to jurisdictional claims in published maps and institutional affiliations.

Supplementary information The online version contains supplementary material available at https://doi.org/10.1038/s41398-021-01387-7.

Received: 16 October 2020 Revised: 25 March 2021 Accepted: 14 April 2021 Published online: 17 May 2021

\section{References}

1. Jalal, $\mathrm{H}$. et al. Changing dynamics of the drug overdose epidemic in the United States from 1979 through 2016. Science 361, eaau1 184 (2018)..

2. NIDA. Overdose Death Rates. https:/wwww.drugabuse.gov/drug-topics/trendsstatistics/overdose-death-rates. (2018).

3. Rawson, R. A. Current research on the epidemiology, medical and psychiatric effects, and treatment of methamphetamine use. J. Food Drug Anal. 21, S77-S81 (2013).

4. Courtney, K. E. \& Ray, L. A. Methamphetamine: an update on epidemiology, pharmacology, clinical phenomenology, and treatment literature. Drug Alcohol Depend. 143, 11-21 (2014).

5. Karila, L. et al. Pharmacological approaches to methamphetamine dependence: a focused review. Br. J. Clin. Pharm. 69, 578-592 (2010).

6. Logan, B. K. Methamphetamine-effects on human performance and behavior. Forensic Sci. Rev. 14, 133-151 (2002).

7. Stock, A. K., Radle, M. \& Beste, C. Methamphetamine-associated difficulties in cognitive control allocation may normalize after prolonged abstinence. Prog. Neuropsychopharmacol. Biol. Psychiatry 88, 41-52 (2019).

8. Rusyniak, D. E. Neurologic manifestations of chronic methamphetamine abuse. Neurol. Clin. 29, 641-655 (2011).

9. Brecht, M. L., Lovinger, K, Herbeck, D. M. \& Urada, D. Patterns of treatment utilization and methamphetamine use during first 10 years after methamphetamine initiation. J. Subst. Abus. Treat. 44, 548-556 (2013).

10. UNODC. World Drug Report. https:/wdr.unodc.org/wdr2020/index.html (2020).

11. Jang, E. Y. et al. The role of reactive oxygen species in methamphetamine selfadministration and dopamine release in the nucleus accumbens. Addict. Biol. 22, 1304-1315 (2017).
12. Najera, J. A. et al. Methamphetamine abuse affects gene expression in brainderived microglia of SIV-infected macaques to enhance inflammation and promote virus targets. BMC Immunol. 17, 7 (2016).

13. Yamamoto, B. K. \& Raudensky, J. The role of oxidative stress, metabolic compromise, and inflammation in neuronal injury produced by amphetamine-related drugs of abuse. J. Neuroimmune Pharm. 3, 203-217 (2008).

14. Stephans, S. E., Whittingham, T. S., Douglas, A. J., Lust, W. D. \& Yamamoto, B. K. Substrates of energy metabolism attenuate methamphetamine-induced neurotoxicity in striatum. J. Neurochem. 71, 613-621 (1998).

15. Wang, G. J. et al. Partial recovery of brain metabolism in methamphetamine abusers after protracted abstinence. Am. J. Psychiatry 161, 242-248 (2004).

16. Volkow, N. D. et al. Recovery of dopamine transporters with methamphetamine detoxification is not linked to changes in dopamine release. Neuroimage 121, 20-28 (2015).

17. Sekine, Y. et al. Methamphetamine causes microglial activation in the brains of human abusers. J. Neurosci. 28, 5756-5761 (2008).

18. Young, E. J., Briggs, S. B. \& Miller, C. A. The actin cytoskeleton as a therapeutic target for the prevention of relapse to methamphetamine use. CNS Neurol. Disord. Drug Targets 14, 731-737 (2015).

19. Moussawi, K. \& Kalivas, P. W. Group II metabotropic glutamate receptors (mGlu2/3) in drug addiction. Eur. J. Pharm. 639, 115-122 (2010).

20. Jayanthi, $S$. et al. Methamphetamine downregulates striatal glutamate receptors via diverse epigenetic mechanisms. Biol. Psychiatry 76, 47-56 (2014).

21. Krasnova, I. N., Justinova, Z. \& Cadet, J. L. Methamphetamine addiction: involvement of CREB and neuroinflammatory signaling pathways. Psychopharmacology 233, 1945-1962 (2016).

22. Ernst, T., Chang, L., Leonido-Yee, M. \& Speck, O. Evidence for long-term neurotoxicity associated with methamphetamine abuse: a 1H MRS study. Neurology 54, 1344-1349 (2000).

23. Chan, P. et al. Rapid ATP loss caused by methamphetamine in the mouse striatum: relationship between energy impairment and dopaminergic neurotoxicity. J. Neurochem. 62, 2484-2487 (1994).

24. Ruan, Q. T. et al. A mutation in Hnrnph1 that decreases methamphetamineinduced reinforcement, reward, and dopamine release and increases synaptosomal hnRNP H and mitochondrial proteins. J. Neurosci. 40, 107-130 (2020).

25. Sassone, J. et al. The synaptic function of parkin. Brain 140, 2265-2272 (2017)

26. Seirafi, M., Kozlov, G. \& Gehring, K. Parkin structure and function. FEBS J. 282, 2076-2088 (2015)

27. Jiang, $\mathrm{H}$. et al. Parkin controls dopamine utilization in human midbrain dopaminergic neurons derived from induced pluripotent stem cells. Nat Commun. 3, 668 (2012).

28. Moszczynska, A. et al. Parkin disrupts the alpha-synuclein/dopamine transporter interaction: consequences toward dopamine-induced toxicity. J. Mol. Neurosci. 32, 217-227 (2007).

29. Huynh, D. P., Scoles, D. R., Ho, T. H., Del Bigio, M. R. \& Pulst, S. M. Parkin is associated with actin filaments in neuronal and nonneural cells. Ann. Neurol. 48, 737-744 (2000).

30. Ren, Y., Zhao, J. \& Feng, J. Parkin binds to alpha/beta tubulin and increases their ubiquitination and degradation. J. Neurosci. 23, 3316-3324 (2003).

31. Cremer, J. N. et al. Changes in the expression of neurotransmitter receptors in Parkin and DJ-1 knockout mice-a quantitative multireceptor study. Neuroscience 311, 539-551 (2015).

32. Everitt, B. J. \& Robbins, T. W. Drug addiction: updating actions to habits to compulsions ten years on. Annu. Rev. Psychol. 67, 23-50 (2016).

33. Salgado, S. \& Kaplitt, M. G. The nucleus accumbens: a comprehensive review. Stereotact. Funct. Neurosurg. 93, 75-93 (2015).

34. Edwards, S. \& Koob, G. F. Escalation of drug self-administration as a hallmark of persistent addiction liability. Behav. Pharm. 24, 356-362 (2013).

35. Rogers, J. L., De Santis, S. \& See, R. E. Extended methamphetamine selfadministration enhances reinstatement of drug seeking and impairs novel object recognition in rats. Psychopharmacology 199, 615-624 (2008).

36. Liu, B., Traini, R., Killinger, B., Schneider, B. \& Moszczynska, A. Overexpression of parkin in the rat nigrostriatal dopamine system protects against methamphetamine neurotoxicity. Exp. Neurol. 359-372 (2013).

37. Gemechu, J. M. et al. Characterization of dopaminergic system in the striatum of young adult Park2(-/) knockout rats. Sci. Rep. 8, 1517 (2018).

38. Bardo, M. T. \& Bevins, R. A. Conditioned place preference: what does it add to our preclinical understanding of drug reward? Psychopharmacology 153, 31-43 (2000). 
39. Moszczynska, A. \& Yamamoto, B. K. Methamphetamine oxidatively damages parkin and decreases the activity of 265 proteasome in vivo. J. Neurochem. 116, 1005-1017 (2011).

40. Everitt, B. J. \& Robbins, T. W. Neural systems of reinforcement for drug addiction: from actions to habits to compulsion. Nat. Neurosci. 8, 1481-1489 (2005).

41. Scofield, M. D. et al. The nucleus accumbens: mechanisms of addiction across drug classes reflect the importance of glutamate homeostasis. Pharm. Rev. $\mathbf{6 8}$ 816-871 (2016)

42. Wise, R. A. Dopamine, learning and motivation. Nat. Rev. Neurosci. 5, 483-494 (2004).

43. Di Chiara, G. Nucleus accumbens shell and core dopamine: differential role in behavior and addiction. Behav. Brain Res. 137, 75-114 (2002).

44. Whitaker, L. R. et al. Associative learning drives the formation of silent synapses in neuronal ensembles of the nucleus accumbens. Biol. Psychiatry $\mathbf{8 0}, \mathbf{2 4 6 - 2 5 6}$ (2016).

45. Gale, J. T., Shields, D. C., Ishizawa, Y. \& Eskandar, E. N. Reward and reinforcement activity in the nucleus accumbens during learning. Front Behav. Neurosci. 8, 114 (2014).

46. Mannella, F., Gurney, K. \& Baldassarre, G. The nucleus accumbens as a nexus between values and goals in goal-directed behavior: a review and a new hypothesis. Front. Behav. Neurosci. 7, 135 (2013).

47. Schultz, W., Apicella, P., Scarnati, E. \& Ljungberg, T. Neuronal activity in monkey ventral striatum related to the expectation of reward. J. Neurosci. 12, 4595-4610 (1992).

48. Goto, Y. \& Grace, A. A. Dopaminergic modulation of limbic and cortical drive of nucleus accumbens in goal-directed behavior. Nat. Neurosci. 8, 805-812 (2005).

49. Khamassi, M. \& Humphries, M. D. Integrating cortico-limbic-basal ganglia architectures for learning model-based and model-free navigation strategies. Front. Behav. Neurosci. 6, 79 (2012).

50. Yin, H. H. \& Knowlton, B. J. The role of the basal ganglia in habit formation. Nat. Rev. Neurosci. 7, 464-476 (2006).

51. Yin, H. H., Ostlund, S. B., Knowlton, B. J. \& Balleine, B. W. The role of the dorsomedial striatum in instrumental conditioning. Eur. J. Neurosci. 22, 513-523 (2005).

52. Yin, H. H., Knowlton, B. J. \& Balleine, B. W. Blockade of NMDA receptors in the dorsomedial striatum prevents action-outcome learning in instrumental conditioning. Eur. J. Neurosci. 22, 505-512 (2005).

53. Yin, H. H., Knowlton, B. J. \& Balleine, B. W. Lesions of dorsolateral striatum preserve outcome expectancy but disrupt habit formation in instrumental learning. Eur. J. Neurosci. 19, 181-189 (2004).

54. Yin, H. H., Knowlton, B. J. \& Balleine, B. W. Inactivation of dorsolateral striatum enhances sensitivity to changes in the action-outcome contingency in instrumental conditioning. Behav. Brain Res. 166, 189-196 (2006).

55. Kreiner, G. Compensatory mechanisms in genetic models of neurodegeneration: are the mice better than humans? Front. Cell Neurosci. 9, 56 (2015).

56. Shimura, $H$. et al. Familial Parkinson disease gene product, parkin, is a ubiquitin-protein ligase. Nat. Genet. 25, 302-305 (2000).

57. Wilhelmus, M. M., Nijland, P. G., Drukarch, B., de Vries, H. E. \& van Horssen, J. Involvement and interplay of Parkin, PINK1, and DJ1 in neurodegenerative and neuroinflammatory disorders. Free Radic.Biol. Med. 53, 983-992 (2012).

58. Frank-Cannon, T. C. et al. Parkin deficiency increases vulnerability to inflammation-related nigral degeneration. J. Neurosci. 28, 10825-10834 (2008).

59. Dawson, T. M. \& Dawson, V. L. The role of parkin in familial and sporadic Parkinson's disease. Mov. Disord. 25(Suppl 1), S32-S39 (2010).

60. Shires, S. E., Kitsis, R. N. \& Gustafsson, A. B. Beyond mitophagy: the diversity and complexity of parkin function. Circ. Res. 120, 1234-1236 (2017).

61. Sandebring, A. A. C.-M., A. Parkin—an E3 ubiquitin ligase with multiple substrates. Alzheimer's Dis. Parkinsonism S10:002, (2012).
62. Martinez, A. et al. Quantitative proteomic analysis of Parkin substrates in Drosophila neurons. Mol. Neurodegener. 12, 29 (2017).

63. Oyama, G. et al. Impaired in vivo dopamine release in parkin knockout mice Brain Res. 1352, 214-222 (2010).

64. Kitada, $T$. et al. Impaired dopamine release and synaptic plasticity in the striatum of parkin-/- mice. J. Neurochem. 110, 613-621 (2009).

65. Zhu, X. R. et al. Non-motor behavioural impairments in parkin-deficient mice. Eur. J. Neurosci. 26, 1902-1911 (2007).

66. Menendez, J. et al. Suppression of Parkin enhances nigrostriatal and motor neuron lesion in mice over-expressing human-mutated tau protein. Hum. Mol. Genet. 15, 2045-2058 (2006).

67. Jiang, H., Jiang, Q. \& Feng, J. Parkin increases dopamine uptake by enhancing the cell surface expression of dopamine transporter. J. Biol. Chem. 279 54380-54386 (2004)

68. Rial, D. et al. Behavioral phenotyping of Parkin-deficient mice: looking for early preclinical features of Parkinson's disease. PLOS ONE 9, e114216 (2014).

69. Lominac, K. D. et al. Mesocorticolimbic monoamine correlates of methamphetamine sensitization and motivation. Front. Syst. Neurosci. 8, 70 (2014).

70. London, E. D. Impulsivity, stimulant abuse, and dopamine receptor signaling. Adv. Pharm. 76, 67-84 (2016).

71. Baik, J. H. Dopamine signaling in reward-related behaviors. Front. Neural Circuits 7, 152 (2013).

72. Arias-Carrion, $\mathrm{O}$. et al. Orquestic regulation of neurotransmitters on rewardseeking behavior. Int. Arch. Med. 7, 29 (2014).

73. Kim, J. H., Austin, J. D., Tanabe, L., Creekmore, E. \& Vezina, P. Activation of group II mGlu receptors blocks the enhanced drug taking induced by previous exposure to amphetamine. Eur. J. Neurosci. 21, 295-300 (2005).

74. Karler, R., Calder, L. D., Chaudhry, I. A. \& Turkanis, S. A. Blockade of "reverse tolerance" to cocaine and amphetamine by MK-801. Life Sci. 45, 599-606 (1989).

75. Wirtshafter, D. \& Stratford, T. R. Evidence for motivational effects elicited by activation of GABA-A or dopamine receptors in the nucleus accumbens shell. Pharm. Biochem. Behav. 96, 342-346 (2010).

76. Ranaldi, R. \& Poeggel, K. Baclofen decreases methamphetamine selfadministration in rats. Neuroreport 13, 1107-1110 (2002).

77. Voigt, R. M., Herrold, A. A., Riddle, J. L. \& Napier, T. C. Administration of GABA(B) receptor positive allosteric modulators inhibit the expression of previously established methamphetamine-induced conditioned place preference. Behav. Brain Res. 216, 419-423 (2011).

78. Snider, S. E., Hendrick, E. S. \& Beardsley, P. M. Glial cell modulators attenuate methamphetamine self-administration in the rat. Eur. J. Pharm. 701, 124-130 (2013).

79. Worley, M. J., Swanson, A. N., Heinzerling, K. G., Roche, D. J. \& Shoptaw, S Ibudilast attenuates subjective effects of methamphetamine in a placebo-controlled inpatient study. Drug Alcohol Depend. 162, 245-250 (2016).

80. Saika, F. et al. Upregulation of CCL7 and CCL2 in reward system mediated through dopamine D1 receptor signaling underlies methamphetamineinduced place preference in mice. Neurosci. Lett. 665, 33-37 (2018).

81. de Guglielmo, G. et al. Increases in compulsivity, inflammation, and neural injury in HIV transgenic rats with escalated methamphetamine self-administration under extended-access conditions. Brain Res. 1726 146502 (2020).

82. Koob, G. F. \& Volkow, N. D. Neurobiology of addiction: a neurocircuitry analysis. Lancet Psychiatry 3, 760-773 (2016).

83. Caulfield, T. R., Fiesel, F. C. \& Springer, W. Activation of the E3 ubiquitin ligase Parkin. Biochem. Soc. Trans. 43, 269-274 (2015).

84. Chakraborty, J., Basso, V. \& Ziviani, E. Post translational modification of Parkin Biol. Direct 12, 6 (2017). 